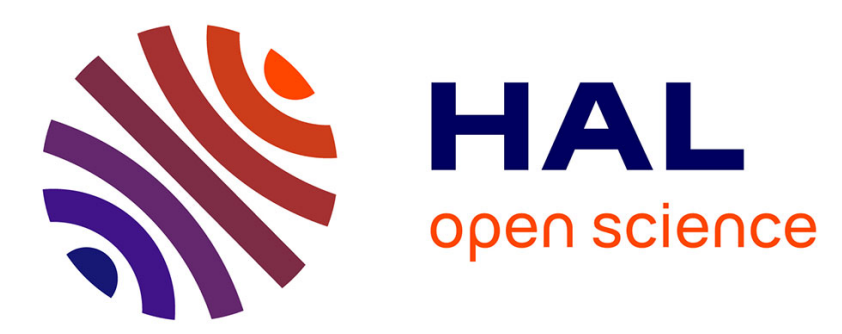

\title{
Inference methods for gas/surface interaction models: from deterministic approaches to Bayesian techniques
}

Anabel del Val, Olivier Le Maitre, Olivier Chazot, Pietro Marco Congedo, Thierry E. Magin

\section{- To cite this version:}

Anabel del Val, Olivier Le Maitre, Olivier Chazot, Pietro Marco Congedo, Thierry E. Magin. Inference methods for gas/surface interaction models: from deterministic approaches to Bayesian techniques. UQOP 2020 - International Conference on Uncertainty Quantification \& Optimisation, UTOPIAE, Nov 2020, Brussels, Belgium. hal-03081323

HAL Id: hal-03081323

https://hal.inria.fr/hal-03081323

Submitted on 18 Dec 2020

HAL is a multi-disciplinary open access archive for the deposit and dissemination of scientific research documents, whether they are published or not. The documents may come from teaching and research institutions in France or abroad, or from public or private research centers.
L'archive ouverte pluridisciplinaire HAL, est destinée au dépôt et à la diffusion de documents scientifiques de niveau recherche, publiés ou non, émanant des établissements d'enseignement et de recherche français ou étrangers, des laboratoires publics ou privés. 


\title{
Inference methods for gas/surface interaction models: from deterministic approaches to Bayesian techniques
}

\author{
Anabel del Val ${ }^{1,2}$, Olivier P. Le Maître ${ }^{3}$, Olivier Chazot $^{1}$, Pietro M. Congedo ${ }^{2}$, \\ and Thierry E. Magin ${ }^{1}$ \\ 1 Aeronautics and Aerospace Department, von Karman Institute for Fluid Dynamics, \\ 1640 Rhode-St-Genèse, Belgium \\ 2 INRIA, Centre de Mathématiques Appliquées, École Polytechnique, Institut \\ Polytechnique de Paris, 91128 Palaiseau, France \\ 3 CNRS, Centre de Mathématiques Appliquées, INRIA, École Polytechnique, Institut \\ Polytechnique de Paris, 91128 Palaiseau, France
}

\begin{abstract}
In this work we review selected experiments and inference methods for the determination of atmospheric entry gas/surface interaction models for air catalysis and nitrogen ablation. Accurate prediction of the gas/surface interaction during spacecraft reentry remains a challenging problem for thermal protection system design. Attempts to model the surface chemistry of catalytic and ablative materials must account for experimental and model uncertainties. We review two sets of experiments and models adopted in the relevant literature for the rebuilding of catalytic properties and nitridation reaction efficiencies. The review is enriched with new perspectives to these problems by using dedicated Bayesian methods.
\end{abstract}

Keywords: Atmospheric entry · Catalysis · Ablation · Bayesian methods

\section{Introduction}

Space travel, since its beginnings in Low Earth Orbit (LEO) to the exploration of our Solar System, has led to countless scientific advancements in what it is one of the most challenging undertakings of humankind. Venturing into Space requires large amounts of kinetic and potential energy to reach orbital and interplanetary velocities. All this amount of energy is dissipated when a space vehicle enters dense planetary atmospheres [24]. The bulk of this energy is exchanged during the entry phase by converting the kinetic energy of the vehicle into thermal energy in the surrounding atmosphere through the formation of a strong bow shock ahead of the vehicle [1]. The interaction between the dissociated gas and the protection system is governed by the material behavior which either acts as a catalyst for recombination reactions of the atomic species in the surrounding gas mixture [9] or ablates as a consequence of the reactive gas mixture that surrounds it, injecting new species into the boundary layer [11]. Characterizing 
both phenomena for different atmospheric and material compositions is quite challenging due to the coupling mechanisms between material surface properties and the resulting ablation and heating rates. All these elements are also tightly coupled to the flowfield computations [30]. A key element of the modeling behind such phenomena is the heterogeneous chemical processes that are taken into account to explain the experiments. It is not always straightforward, from a priori knowledge point of view, to know which chemical processes underpin the macroscopic effects we see in thermal protection materials subjected to a reactive flow environment.

All the above makes the determination of gas/surface interactions of thermal protection materials a complex task subjected to experimental and model uncertainties. The design and performance of atmospheric entry vehicles must account for these uncertain characterizations. It is relatively common when dealing with complex physical phenomena to resort to simple, non-intrusive a priori forward uncertainty propagation techniques [36]. These techniques assume a priori probability distributions for the main model parameters. Sensitivity analyses are then performed to discriminate the important ones. They also assume that the exact value is sufficiently well known and within the considered uncertainty range. These methods do not use any experimental observation to calibrate such parameters. The interest of using experimental information is that it leads to objective uncertainty levels and provides likely values rather than a priori guesses, achieving better and more reliable predictions. The calibration from experimental data focuses on a Bayesian approach that has the advantage of providing a complete objective characterization of the parameters' uncertainty through their resulting posterior distribution.

In this context, knowing how the experimental procedure is carried out is fundamental for the formulation of the inference method. Current plasma wind tunnel experiments rely heavily on the accurate characterization of free stream conditions that serve as input to Computational Fluid Dynamics (CFD) models. In the particular case of catalytic materials, this step proves critical to correctly account for the catalytic heat flux. Another important aspect of high temperature flow testing is that even though our interest lays in the heterogeneous chemical processes, it is common to have additional parameters for which direct experimental observations are not available. These parameters are needed to perform the inference but we are not explicitly interested in getting their distributions. Traditional Bayesian approaches deal with this problem by prescribing prior distributions on such parameters at the expense of some of the observations consumed to evaluate these nuisance parameter posteriors. Consequently, it is important to remark their impact on the quality of the inference [38]. These challenges are most critical for catalytic materials. Ablative materials have a distinct behavior under such high temperature reacting flows. The fact that they undergo ablation, i.e. the chemical consumption of the material, generates a visible surface recession that can be measured and gives direct evidence of the chemical processes involved in such phenomena. The main issue with ablative materials is centered on the fact that while an overall response is measured (re- 
cession), it is difficult to isolate the different effects that happen on the surface to produce the macroscopic response. Both systems present challenges for the efficient inference of heterogeneous chemical parameters. In this paper we review the traditional methods for the deterministic rebuilding of such parameters and describe the proposed inferences using Bayesian methods and how the aforementioned challenges are addressed. The Bayesian methods exploit the experimental data resulting from measurements performed by Panerai [29] and Helber [17] on two different types of materials: ceramic matrix composites and graphite, respectively. While deterministic approaches require more data and assumptions to extract the model parameters from the experimental data, Bayesian methods offer a complete characterization of their uncertainty. This work paves the way to a new design of experiments paradigm within the Thermal Protection System (TPS) community, where the most informative experiments will be sought.

The article is organized as follows: Sec. 2 addresses the sets of experiments dedicated to the study of catalytic and ablative materials reviewed here. Sec. 3 reviews the models used for the deterministic approaches and how they are used together with the experimental data to rebuild the model parameters. Sec. 4 showcases the Bayesian methods proposed and Sec. 5 discusses the conclusions and outlook.

\section{Plasma wind tunnel experiments}

In this section, we review the different experimental procedures adopted for the study of catalytic and ablative materials at the von Karman Institute (VKI). The experimental data is consequently used to rebuild different model parameters that define the gas/surface interaction of the different materials in question.

\subsection{Heterogeneous catalysis}

We consider the experimental set-up of the Plasmatron facility at VKI, an Inductively-Coupled Plasma (ICP) wind tunnel [5]. The plasma flow is generated by the induction of electromagnetic currents within the testing gas in the plasma torch; this process creates a high-purity plasma flow which leaves the testing chamber through the exhaust.

As a simple model of TPS response, we define the catalytic coefficient $\gamma$ as the ratio of the number of atoms that recombine on the material surface over the total number of atoms that hit it. We assume the same recombination probability for the nitrogen and oxygen species constituting the air plasma, leading to just one single catalytic parameter to characterize the material under atmospheric entry conditions. In a typical experiment, one sequentially exposes two probes to the plasma flow: a reference probe made of a well-known material (copper) with a catalytic parameter $\gamma_{\text {ref }}$, and a test probe which holds a sample of the TPS material with the unknown catalytic coefficient $\gamma_{\text {TPS }}$ to be inferred. The following instruments equip the Plasmatron. For pressures, a water-cooled Pitot probe measures the dynamic pressure $P_{\mathrm{d}}$ within the plasma jet, and an absolute 
pressure transducer records the static pressure $P_{\mathrm{s}}$ in the Plasmatron chamber. The reference probe is an hemispherical device (25 $\mathrm{mm}$ radius) equipped with a water-cooled copper calorimeter at the center of its front face. The calorimeter has a cooling water system that maintains the surface temperature of the reference probe. The reference probe heat flux is deduced from the mass flow (controlled by a calibrated rotameter) circulating in the cooling system and the inlet/outlet water temperature difference measured by thermocouples as a result of the exposure to the plasma flow. For the test probes, we measure directly the emissivities $\epsilon$ and the surface temperatures $T_{\mathrm{w}}$. The determination of the heat flux assumes a radiative equilibrium at the surface, with the relation $q_{\mathrm{w}}=\sigma \epsilon T_{\mathrm{w}}^{4}$, where $\sigma$ is the Stefan-Boltzmann constant, $\epsilon$ is the emissivity measured with an infrared radiometer, and $T_{\mathrm{w}}$ is the wall temperature which is measured using a pyrometer. More details on how these measuring devices work can be found in [28]. Figure 1 schematizes the Plasmatron and its instrumentation for catalytic property determination.

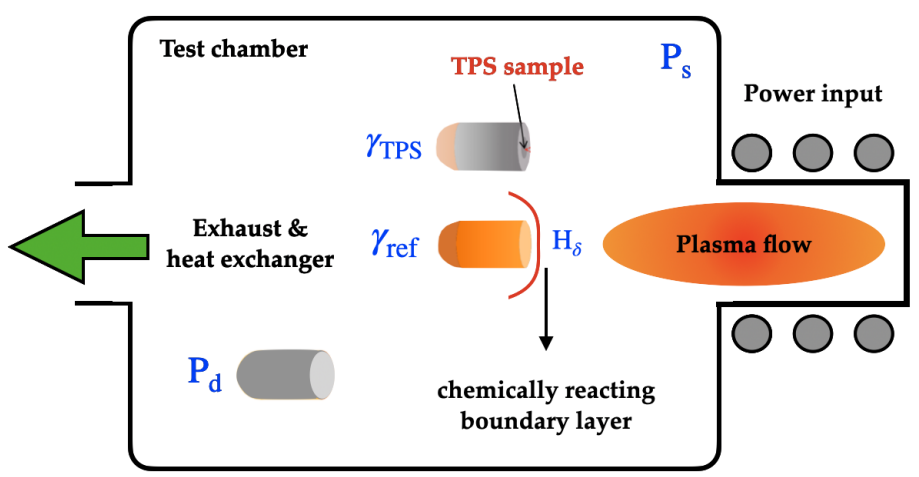

Fig. 1. Schematic view of the Plasmatron experimental set-up.

The underlying idea of the experimental procedure is to perform first measurements of the wall temperature, heat flux and pressures $P_{\mathrm{d}}$ and $P_{\mathrm{s}}$ with the reference probe set in the plasma jet. As these measurements depend on the state of the free stream flow, in particular on the enthalpy $H_{\delta}$ at the boundary layer edge $\delta$, the free stream conditions can be deduced if one knows the contribution to the heat flux of the surface catalysis. In our formulation, this is equivalent to knowing the catalytic coefficient $\gamma_{\text {ref }}$ of the reference probe. Then, in a second stage, the test probe is set in place of the reference probe in the plasma jet. The corresponding steady state wall temperature $T_{\mathrm{w}}$ and emissivity $\epsilon$ are measured and, assuming that the free stream flow conditions have not changed, the catalytic coefficient $\gamma_{\text {TPS }}$ of the test probe can be inferred. 


\subsection{Thermochemical ablation}

The experimental set-up installed at the Plasmatron facility offers intrusive and non-intrusive (optical) measurement techniques. The instrumentation equipment consists of a video camera, a 2-color pyrometer, a broad-band radiometer, a digital camera, and a spectrometer with intensified Charge-Coupled Device (CCD). The main data of interest during the investigation reported here is surface ablation rates of graphite in a pure nitrogen plasma $\dot{s}$ and measurements of the locally resolved ablation product cyanogen $(\mathrm{CN})$ boundary layer emission and densities $\rho_{\mathrm{CN}}$. These measurements are directly linked to the model parameter we want to rebuild, the nitridation reaction efficiency $\gamma_{\mathrm{N}}^{\mathrm{CN}}$.

The same equipment as for the study of heterogeneous catalysis is used here for the determination of the free stream conditions through the use of heat flux measurements on a reference (copper) material. A set of other dedicated measurements are used for the characterization of the ablative material itself. The total stagnation point recession $\dot{s}$ is measured using a digital camera (Nikon D5000) attached to a $400 \mathrm{~mm}$ lens, giving a resolution of about $0.03 \mathrm{~mm}$ per pixel. The test sample is pre-heated (cleaned and dried) by the Argon plasma used to start the Plasmatron facility. After starting the plasma on Argon gas, with the test sample in place, the test gas is switched to pure nitrogen. The stagnation point of the test sample is placed $445 \mathrm{~mm}$ from the torch exit. The strong radiative signature of the $\mathrm{CN}$ molecule allows for its easy probing by emission spectroscopy via the strong violet system emission [31][6]. One can further benefit from the low surface recession rate of the graphite sample, contrary to air ablation, allowing to average several recorded spectra per ablation test. The emission spectroscopy setup consists of an Acton Series SP-2750 spectrograph of $75 \mathrm{~cm}$ focal length combined with an ICCD PI-MAX camera with a frame of 1024 $\times 1024$ pixels. The two- dimensional ICCD array enables spectral measurements across the complete plasma jet in an imaged plane of $20 \mathrm{~cm}$, yielding a spatial resolution of $0.195 \mathrm{~mm}$. For each acquisition, the camera records a data matrix with wavelength distributed along the horizontal axis and the lateral positions of the observed plasma radius distributed along the vertical axis.

Objective of the spectral measurements is the determination of the locally resolved $\mathrm{CN}$ emission and the experimental $\mathrm{CN}$ species density $\rho_{\mathrm{CN}}$. But as the plasma jet is being observed from the side, the recorded signal is a result of the local emission integrated along the line-of-sight, projected onto the ICCD sensor. If we assume axisymmetry of the jet and an optically thin gas, the inverse Abel transformation provides the local emission coefficient at distance $r$ from the center. Treatment of the experimental spectra is necessary prior to conversion of local emission. More details about the experimental CN species density determination can be found in [17]. 
del Val et al.

\section{Deterministic approaches to the inference of model parameters}

In this section, we highlight the different approaches adopted in the relevant literature to rebuild model parameters deterministically and learn about gas/surface interaction phenomena through experiments. We also make the split between catalytic and ablative materials due to the different approaches to the rebuilding and the models.

\subsection{Heterogeneous catalysis}

The catalytic activity on a material surface cannot be measured directly. We need models and simulations to bypass that lack of knowledge and use other measurable quantities to rebuild the model parameter we are seeking. Modelbased numerical simulations include the catalytic recombination parameter $\gamma$ in their model to account for this phenomenon on the computation or prediction of relevant quantities. These methods use experiments and models intertwined in a complex fashion. To identify the TPS catalytic properties $\gamma_{\mathrm{TPS}}$, chemically reacting boundary layer simulations are employed in the vicinity of the stagnation point [1]. The Boundary Layer (BL) code implements the Navier-Stokes equations. To solve the system, closure models for the thermodynamic and transport properties as well as the chemical production terms of the different species are needed. Transport fluxes are derived from kinetic theory using the ChapmanEnskog method for the solution of the Boltzmann equation [12,26]. Diffusion fluxes are computed through the generalized Stefan-Maxwell equations $[7,13$, 21]. For the homogeneous chemistry, the Law of Mass Action is used to compute production rates as proportional to the product of the reactant densities raised to their stoichiometric coefficients [22]. The thermodynamic properties, such as the enthalpy, are derived from statistical mechanics $[1,37]$ for a reacting mixture of perfect gases, assuming thermal equilibrium and chemical non-equilibrium. The dependency of the catalytic parameters comes from the boundary condition where this term accounts for the recombination efficiency at the wall. Apart from the closure models, the parabolic nature of the BL model requires the imposition of two boundary conditions: the external flow conditions at the boundary layer edge [23], and at the material surface where recombination reactions can be triggered depending on the catalytic nature of such material [14]. More details about the derivation, coordinate transformations and numerical implementation of the BL code are available in the work of Barbante [2].

In summary, the predictive quantity of the model is the wall heat flux

$$
q_{\mathrm{w}}=q_{\mathrm{w}}\left(\gamma, T_{\mathrm{w}}, P_{\delta}, H_{\delta}, \delta, \frac{\partial u_{\delta}}{\partial x}, v_{\delta} \frac{\partial}{\partial y}\left(\frac{\partial u_{\delta}}{\partial x}\right)\right),
$$

which depends on the free stream conditions (subscript $\delta$ ), the thickness of the boundary layer $\delta$, the catalytic parameter of the material $\gamma$ and the surface temperature $T_{\mathrm{w}}$. An auxiliary 2D magnetohydrodynamic axisymmetric simulation 
assuming Local Thermodynamic Equilibrium (LTE) is performed to simulate the torch and the chamber of the wind tunnel [23]. Relaying on the knowledge of the operating conditions of the Plasmatron, such as electric power, injected mass flow, static pressure and probe geometry, this 2D simulation lets us compute non-dimensional parameters that define the momentum influx to the boundary layer (interested reader is directed to [10]). The prediction we are seeking to match the experimental data is now recast as

$$
q_{\mathrm{w}}=q_{\mathrm{w}}\left(\gamma, T_{\mathrm{w}}, P_{\delta}, H_{\delta}, \Pi_{1}, \Pi_{2}, \Pi_{3}\right),
$$

where $\Pi_{1, \ldots, 3}$ are the non-dimensional parameters.

We solve this equation iteratively over the outer edge temperature $T_{\delta}$, which is directly linked to $H_{\delta}$, until the numerical heat flux matches the one measured experimentally with the calorimetric probe. The procedure returns the enthalpy and velocity gradient at the boundary layer outer edge. Once the plasma enthalpy has been determined, we can run the BL code for various combinations of material catalysis and temperature, to obtain a heat flux abacus, $q_{\mathrm{w}}=q_{\mathrm{w}}\left(T_{\mathrm{w}}, \gamma\right)$. The abacus defines a chemically reacting frame for one enthalpy, pressure and model geometry combination. The TPS catalysis can be determined by identification of the $\gamma$ contour, where the actual experimental conditions $\left(q_{\mathrm{w}}, T_{\mathrm{w}}\right)$ lay.

\subsection{Thermochemical ablation}

The approach here presented is also based on a full Navier-Stokes solution with boundary conditions for surface mass and energy balances. This approach has been widely used to study the ablative gas/surface interaction of TPS with the surrounding flowfield $[4,35,8]$. While the gas phase is governed by the NavierStokes equations, the surface is not fully simulated, but is approximately modeled. The plasma boundary layer edge condition composed of the temperature $T_{\delta}$, axial velocity $v_{\delta}$, and species densities $\rho_{i, \delta}$, dependent on the temperature and static pressure $P_{\mathrm{s}}$, is fully described by the experimental-numerical plasma rebuilding procedure described previously in the context of heterogeneous catalysis. Quantities at the boundary layer edge serve as input to a 1D stagnation-line code with ablative boundary condition [34], with the goal to numerically simulate the ablation experiments. A set of balance equations are solved with respect to the conserved quantities of the gas, i.e., mass, momentum and energy, which are imposed as boundary values for the Navier-Stokes equations. For the ablation experiments considered in this work, only a mass balance equation is needed given that we invoke the no slip condition for the momentum equations and the wall temperature is imposed as it is measured. This balance is obtained limiting the control volume of the mass conservation equation to the thin lamina representing the gas-surface interface $[18,33]$. The unknown nitridation reaction efficiency is recovered from the closure model considered for the surface mass balance (find the derivation in more detail in [17]):

$$
\gamma_{\mathrm{N}}^{\mathrm{CN}}\left(T_{\mathrm{w}}\right)=\frac{\dot{m}_{s}}{\rho_{\mathrm{N}_{\mathrm{w}}}} \frac{\mathcal{M}_{\mathrm{CN}}}{\mathcal{M}_{\mathrm{C}}}\left(\frac{\mathcal{R} T_{\mathrm{w}}}{2 \pi \mathcal{M}_{\mathrm{N}}}\right)^{-1 / 2}
$$


where the term $\dot{m}_{\mathrm{s}}$ is the mass loss flux of the ablated material, $\mathcal{M}$ denotes the molar masses of the different species, $T_{\mathrm{w}}$ is the temperature at the wall, which is measured, and $\rho_{\mathrm{N}_{\mathrm{w}}}$ is the nitrogen density at the wall. The mass loss $\dot{m}_{\mathrm{s}}$ is directly linked to the measured recession rate $\dot{\mathrm{s}}$ through the known material density of the graphite $\operatorname{rod} \rho_{\mathrm{s}}=1760 \mathrm{~kg} / \mathrm{m}^{3}$. The unknown $\rho_{\mathrm{N}_{\mathrm{w}}}$ is computed by accounting in a fully-coupled way the effect of the ablation product injection. We point out that this approach does not take any changes in the surface microstructure during ablation into account and is solely based on steady-state ablation. In addition, it is almost impossible to observe changes of the surface state during ablation in the plasma wind tunnel in-situ. It is important to remark the fact that the experimentally derived $\rho_{\mathrm{CN}}$ is only used to validate the rebuilding methodology without using its information to retrieve $\gamma_{\mathrm{N}}^{\mathrm{CN}}$.

\section{Bayesian approaches to the inference of model parameters}

In this section we review the Bayesian formulations proposed for inferring catalytic parameters and nitridation reaction efficiencies from the experiments depicted in Sec. 2. We also report the main results for two different sets of cases, S1 test from Panerai [29] for ceramic matrix composite materials and G4-G7 cases from Helber [17] for graphite ablation.

\subsection{Bayes theorem}

The inference of model parameters uses the Bayes formula which can be generally formulated as

$$
\mathcal{P}(\boldsymbol{q} \mid \mathcal{M})=\frac{\mathcal{L}(\mathcal{M} \mid \boldsymbol{q}) \mathcal{P}(\boldsymbol{q})}{\int_{\Omega} \mathcal{L}(\mathcal{M} \mid \boldsymbol{q}) \mathcal{P}(\boldsymbol{q}) d \boldsymbol{q}},
$$

where $\boldsymbol{q}$ is the generic vector of parameters, having for components the parameters of the analysis, and $\mathcal{M}$ is the vector of the measured quantities used for the analysis. In the present Bayesian setting, $\boldsymbol{q}$ and $\mathcal{M}$ are real-valued random vectors. We denote $\mathcal{P}(\boldsymbol{q})$ the prior probability distribution of the parameters that expresses one's beliefs on possible values of $\boldsymbol{q}$ before the measurements are made available. $\mathcal{L}(\mathcal{M} \mid \boldsymbol{q})$ is the likelihood function, i.e., the probability of observing measurements $\mathcal{M}$ given $\boldsymbol{q}$. Typically, the likelihood compares the measurements with model predictions (functions of $\boldsymbol{q}$ ), and relies on a noise model to account for the measurement error; a model error contribution can also be included [19]. Specifically, the comparison can be made on the raw measurements or more generally on some derived quantities which is the case of our study. From the measurements $\mathcal{M}$, Bayesian inference updates the prior distribution $\mathcal{P}(\boldsymbol{q})$ to the posterior probability distribution $\mathcal{P}(\boldsymbol{q} \mid \mathcal{M})$.

The posterior distribution in Eq. 4 is usually not known in a closed form due to the complexity of the mapping $\boldsymbol{q} \rightarrow M(\boldsymbol{q})$ where $M(\boldsymbol{q})$ is the vector of model predictions using the forward model. The noise and error models can also complicate calculations. Therefore, sampling strategies, such as Markov Chain 
Monte Carlo (MCMC) methods [25], are needed to estimate the statistics of the posterior distribution of $\boldsymbol{q}$ (e.g., mean, moments, median, and mode). In this work, we use the Adaptive Metropolis (AM) algorithm [15], an extension of the Random-Walk Metropolis (RWM) algorithm, which adapts the proposal covariance matrix using previously sampled points. Lastly, the integral under the denominator in Eq. 4 extends to the space of $\boldsymbol{q}$, denoted here with the Greek letter $\Omega$. In practical terms, this integral is called the evidence and it is a single number. It usually does not mean anything by itself, but can be important when comparing different model choices.

\subsection{Heterogeneous catalysis}

For the inference of catalytic parameters we have denoted the set of experimental data as $\mathcal{M}=\left(P_{\mathrm{s}}^{\text {meas }}, P_{\mathrm{d}}^{\text {meas }}, q_{\mathrm{w}}^{\text {ref, TPS, meas }}, T_{\mathrm{w}}^{\text {ref,TPS,meas }}\right)$. The issue with this inference is the fact that the model predictions $q_{\mathrm{w}}=q_{\mathrm{w}}\left(\gamma, T_{\mathrm{w}}, P_{\delta}, H_{\delta}, \Pi_{1}, \Pi_{2}, \Pi_{3}\right)$ are not just functions of the catalytic coefficient $\gamma$, but also depend on all the inputs of the BL code. It is worth noting that the dependencies of the predictions can be recast as $q_{\mathrm{w}}=q_{\mathrm{w}}\left(\gamma, T_{\mathrm{w}}, P_{\mathrm{s}}, H_{\delta}, P_{\mathrm{d}}\right)$ given that the non-dimensional parameters are set for each case and the predictions are barely sensitive to them for the given measurements. The edge pressure is taken as the static pressure of the chamber and the stagnation pressure is used to derive the momentum influx to the boundary layer through the given non-dimensional parameters. As already mentioned, the pressures and wall temperatures are measured in the experiment, but only with limited precision, while the enthalpy $H_{\delta}$ is simply not known. In this Bayesian formulation we propose to infer all model parameters (ref and TPS for reference and protection material) jointly, given that they both play the same role in the inference problem and our level of prior knowledge about both materials can be safely assumed to be the same. Consequently, there may be zero, or multiple, boundary layer edge conditions consistent with the measurements. Since the boundary layer edge conditions can not be completely characterized, the remaining uncertainty should be accounted for when inferring the TPS catalytic coefficient $\gamma_{\text {TPS }}$.

One possibility to handle this issue is to consider the whole set of uncertain quantities, not just the quantities of interest $\gamma_{\text {ref }}$ and $\gamma_{\text {TPS }}$, but also the so-called nuisance parameters. In that case, we have $\boldsymbol{q}=\left(\gamma_{\mathrm{ref}}, \gamma_{\mathrm{TPS}}, T_{\mathrm{w}}^{\mathrm{ref}}, T_{\mathrm{w}}^{\mathrm{TPS}}, P_{\mathrm{s}}, P_{\mathrm{d}}, H_{\delta}\right)$ in the inference problem. The introduction of the nuisance parameters induces several difficulties related to the necessity to specify their prior distributions, the increased dimensionality of the inference space, and the consumption of information for the inference of the nuisance parameters. This last issue is detrimental to learning the parameters of interest. We derive an alternative formulation for the joint inference of the two catalytic coefficients $\gamma=\left(\gamma_{\mathrm{ref}}, \gamma_{\mathrm{TPS}}\right)$. The proposed formulation only depends on the two catalytic coefficients and not on the other nuisance parameters. As a result, only the prior $\mathcal{P}(\gamma)$ is needed. Assuming independent unbiased Gaussian measurement errors, with magnitude $\sigma$, the 
proposed likelihood of $\mathcal{M}$ would read as

$$
\begin{aligned}
\mathcal{L}^{\text {opt }}(\mathcal{M} \mid \gamma)= & \exp \left[-\frac{\left(P_{\mathrm{s}}^{\text {meas }}-P_{\mathrm{s}}^{\mathrm{opt}}(\gamma)\right)^{2}}{2 \sigma_{P_{\mathrm{s}}}^{2}}\right] \exp \left[-\frac{\left(P_{\mathrm{d}}^{\text {meas }}-P_{\mathrm{d}}^{\mathrm{opt}}(\gamma)\right)^{2}}{2 \sigma_{P_{\mathrm{d}}}^{2}}\right] \times \\
& \times \prod_{i \in\{\text { ref,TPS }\}} \exp \left[-\frac{\left(q_{\mathrm{w}}^{i, \text { meas }}-q_{\mathrm{w}}^{i, \text { opt }}(\gamma)\right)^{2}}{2 \sigma_{q_{\mathrm{w}}}^{2}}-\frac{\left(T_{\mathrm{w}}^{i, \text { meas }}-T_{\mathrm{w}}^{i, \text { opt }}(\gamma)\right)^{2}}{2 \sigma_{T_{\mathrm{w}}}^{2}}\right],
\end{aligned}
$$

where the dependence of the optimal values on the two material properties has been made explicit for clarity.

Given $\mathcal{M}$ and a value for the couple of catalytic coefficients, the optimal nuisance parameters and associated heat fluxes are determined using the BL code. The procedure for this optimization is the Nelder-Mead algorithm [27], which is a gradient-free method requiring only evaluations of the BL model solution. Typically, a few hundreds resolutions of the BL model are needed to converge to the optimum of (5). The computational cost of the optimization prevents us from using directly this approach to draw samples of $\gamma$ from their posterior distribution, and this fact motivates the approximation of the optimal (log) likelihood function using a Gaussian Process surrogate [32]. Fig. 2 shows the obtained posterior distributions for experiment S1 reported in [29].

We can observe that the distributions of both $\gamma_{\text {ref }}$ and $\gamma_{\text {TPS }}$ drop to small values at both ends of the spectrum, reducing the support from the prior distributions proposed. This behavior can be explained by the proposed likelihood form, which uses all the available measurements to access the fitness of the model predictions. It is also important to notice that both distributions have well-defined peaks for $\gamma_{\text {ref }} \simeq 0.016$ and $\gamma_{\mathrm{TPS}} \simeq 0.01$. In this framework, no assumptions are made concerning $\gamma_{\text {ref }}$, which is estimated along with the protection material parameter with no differences in their prior knowledge. It can be suggested that a deeper experimental study can provide more insights to the behavior of the reference material and a different prior can be defined for the same analysis where differences in knowledge between the two probes can be then accounted for. The distributions of the optimal nuisance parameters can be obtained by building surrogates on these quantities as functions of $\gamma_{\mathrm{ref}}$ and $\gamma_{\mathrm{TPS}}$. This is done by using the same Gaussian process methods as for the optimal likelihood. The joint posterior of the catalytic parameters can be then propagated through such surrogates to obtain the resulting distributions of the nuisance parameters.

\subsection{Thermochemical ablation}

For the nitridation experiments, we have as the set of measurements $\mathcal{M}=\left(P_{\mathrm{s}}^{\text {meas }}, P_{\mathrm{d}}^{\text {meas }}, T_{\mathrm{w}}^{\text {meas }}, \dot{s}^{\text {meas }}, \rho_{\mathrm{CN}}^{\text {meas }}\right)$. It is important to notice that for a stagnation line simulation in a reacting flow, the inlet boundary condition must comprise species densities $\rho_{\mathrm{i}, \delta}$, temperature $T_{\delta}$, pressure $P_{\delta}$ and velocity components $u_{\delta}, v_{\delta}$, while the surface conditions are temperature at the wall $T_{\mathrm{w}}$ which 


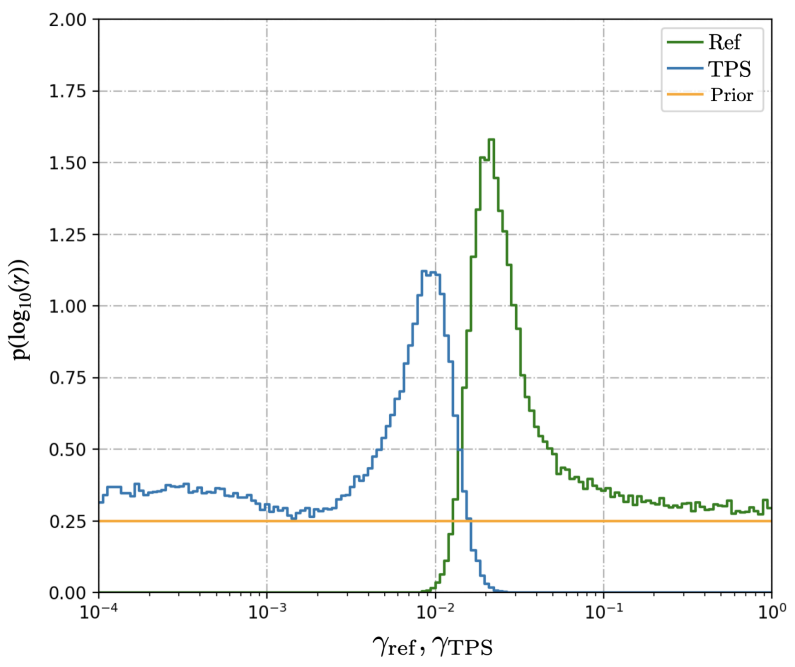

Fig. 2. Marginal posteriors obtained with the Bayesian formulation.

closes the energy equation; we assume no slip condition, which closes the momentum equations and we define surface mass balances for the pseudo-species mass equations. This mass balance needs the chemical mechanism at the surface to be specified and we also need to give values to the different parameters. All in all, we intend to find the flow solutions that are compatible with our experimental observations under the considered model. This means that from the set of observations $\mathcal{M}$ we want to infer the inlet conditions and the wall conditions in such a way that we are left with a population of possible flow solutions. As we work with a mixture of 9 species: $\left\{\mathrm{e}^{-}, \mathrm{C}^{+}, \mathrm{C}_{2}, \mathrm{C}_{3}, \mathrm{CN}, \mathrm{C}, \mathrm{N}, \mathrm{N}^{+}, \mathrm{N}_{2}\right\}$, we are left with a 15 dimensional problem if we only consider nitridation at the wall.

To properly define our vector of parameters to be inferred $\boldsymbol{q}$ we need to take into account some physical relationships. Relaying on the knowledge of the operating conditions of the Plasmatron, such as electric power, injected mass flow, static pressure and probe geometry, we can compute non-dimensional parameters that define the momentum influx to the boundary layer (same parameters as for the catalytic case). These non-dimensional parameters together with the dynamic pressure expression, corrected for viscous effects $P_{\mathrm{d}} / K_{\mathrm{H}}=1 / 2 \rho v^{2}[3]$, let us define a relationship for the inlet velocity components $u_{\delta}, v_{\delta}$ with the measured dynamic pressure. The need to specify two velocity components falls from the spherical coordinates transformation used in the dimensionally-reduced Navier-Stokes equations [20]. Generally, it is safe to assume that we have thermochemical equilibrium at the edge of the boundary layer for these conditions as already studied by Helber [16]. This assumption lets us relate the species densities to the temperature and the pressure, reducing our problem dimensionality further. What the additional physical relations depicted here are doing in prac- 
ticality is to constrain our search for the more likely flow solutions to the ones that have thermo-chemical equilibrium at the inlet, with a particular given relationship between influx velocity components while complying with the dynamic pressure measurements. It is important to mention that the variability of the inlet non-dimensional parameters with the operating conditions is small as shown by Panerai [28] and they can be assumed to play a negligible role in the inference we want to carry out. In any case, it is possible to test these assumptions by getting rid of this additional structure in our model, therefore liberating the search for flow solutions to any with any velocity components. At the end of the analysis, we are left with 5 parameters to calibrate, namely $\boldsymbol{q}=\left(P_{\mathrm{s}}, P_{\mathrm{d}}, T_{\mathrm{w}}, T_{\delta}, \gamma_{\mathrm{N}}^{\mathrm{CN}}\right)$. In the experiment, a previous step is done to measure the free stream condition of the plasma jet $T_{\delta}$, which is needed for the deterministic rebuilding presented in Sec. 3.2. In this Bayesian approach, we manage to get rid of that experimental step because our inference is not sensitive enough to $T_{\delta}$. This is due to the fact that we do not measure heat fluxes here but instead have recession rates and $\mathrm{CN}$ densities. In the Bayesian approach, we can assume ignorance for $T_{\delta}$ and still be able to retrieve what we are looking for, $\gamma_{\mathrm{N}}^{\mathrm{CN}}$. This reduction of observations in our inference problem is an important advantage of the Bayesian approach over the deterministic rebuilding.

Finally, having our set of measurements and parameters defined, we propose a likelihood of the form

$$
\begin{aligned}
& \mathcal{L}\left(\mathcal{M} \mid P_{\mathrm{s}}, P_{\mathrm{d}}, T_{\mathrm{w}}, T_{\delta}, \gamma_{\mathrm{N}}^{\mathrm{CN}}\right) \propto \exp \left[-\frac{\left|\dot{s}^{\text {meas }}-\dot{s}\right|^{2}}{2 \sigma_{\dot{s}}^{2}}\right] \exp \left[-\frac{\left|P_{s}^{\text {meas }}-P_{s}\right|^{2}}{2 \sigma_{P_{\mathrm{s}}}^{2}}\right] \times \\
& \quad \times \exp \left[-\frac{\left|\rho_{C N}^{\mathrm{meas}}-\rho_{C N}\right|^{2}}{2 \sigma_{\rho_{\mathrm{CN}}}^{2}}\right] \exp \left[-\frac{\left|T_{w}^{\text {meas }}-T_{w}\right|^{2}}{2 \sigma_{T_{\mathrm{w}}}^{2}}\right] \exp \left[-\frac{\left|P_{\mathrm{d}}^{\text {meas }}-P_{\mathrm{d}}\right|^{2}}{2 \sigma_{P_{\mathrm{d}}}^{2}}\right],
\end{aligned}
$$

where $\dot{s}=\dot{s}\left(P_{\mathrm{s}}, u_{\delta}, v_{\delta}, T_{\mathrm{w}}, T_{\delta}, \gamma_{\mathrm{N}}^{\mathrm{CN}}\right), \rho_{\mathrm{CN}}=\rho_{\mathrm{CN}}\left(P_{\mathrm{s}}, u_{\delta}, v_{\delta}, T_{\mathrm{w}}, T_{\delta}, \gamma_{\mathrm{N}}^{\mathrm{CN}}\right), u_{\delta}=$ $=u_{\delta}\left(P_{\mathrm{s}}, P_{\mathrm{d}}, T_{\delta}\right)$ and $v_{\delta}=v_{\delta}\left(P_{\mathrm{s}}, P_{\mathrm{d}}, T_{\delta}\right)$. Notice the dependency of $\dot{s}$ and $\rho_{\mathrm{CN}}$ on the velocity components which are functions of the dynamic pressure $P_{\mathrm{d}}$, the static pressure $P_{\mathrm{s}}$ and the temperature $T_{\delta} . P_{\mathrm{s}}$ and $T_{\delta}$ are needed to compute the density of the mixture defined in the equation relating the dynamic pressure to one of the velocity components. The inference is carried out on the constitutive variables $P_{\mathrm{s}}, P_{\mathrm{d}}$ and $T_{\delta}$ while the relationship with the velocity components is computed externally and the velocity components are fed to the model to output $\dot{s}$ and $\rho_{\mathrm{CN}}$. It is simpler to keep the original inputs to the solver which would let us use this framework as it is to asses possible discrepancies of the auxiliary $2 \mathrm{D}$ problem (to obtain the non-dimensional parameters) with the experiments, thereby having to include the velocities in the inference problem in the future. The computation of the model outputs $\dot{s}$ and $\rho_{\mathrm{CN}}$ requires the solution of the 1D stagnation line problem which can be expensive to evaluate for a proper statistical characterization of the posterior distribution. To overcome this issue, $\dot{s}$ and $\rho_{\mathrm{CN}}$ are approximated again by Gaussian Process surrogate models whose evaluations are cheaper. 
We can see in Fig. 3 the different inferences that we carried out when using each piece of information independently and all together. Overall we can see that the calibration seems consistent, the support of the distribution obtained with all measurements is contained in within the support of each of the parts and one can see some information gain (reduced support compared to either of the parts'). Overall, good agreement seems to be found, although we can clearly see that as the wall temperature increases (from G4 to G7), there seems to be an over prediction of $\rho_{\mathrm{CN}}$ that is not fully consistent with the measurements of recession rates. This is reflected on the calibration of $\gamma_{\mathrm{N}}^{\mathrm{CN}}$ as both measurements are directly and greatly affected by it. If we pay attention to case G6 (lower left), we can see that this is not the case. Here, the overlap of support is very small and the distribution that contains the information of both measurements does not share most of its support with the calibration obtained by using only CN densities. It is clear that, in this case, the results represent a trade-off between the two measurements and the physics must be studied further. The issue could be double-fold: either an epistemic uncertainty underlying the physical model or biased experimental observations.
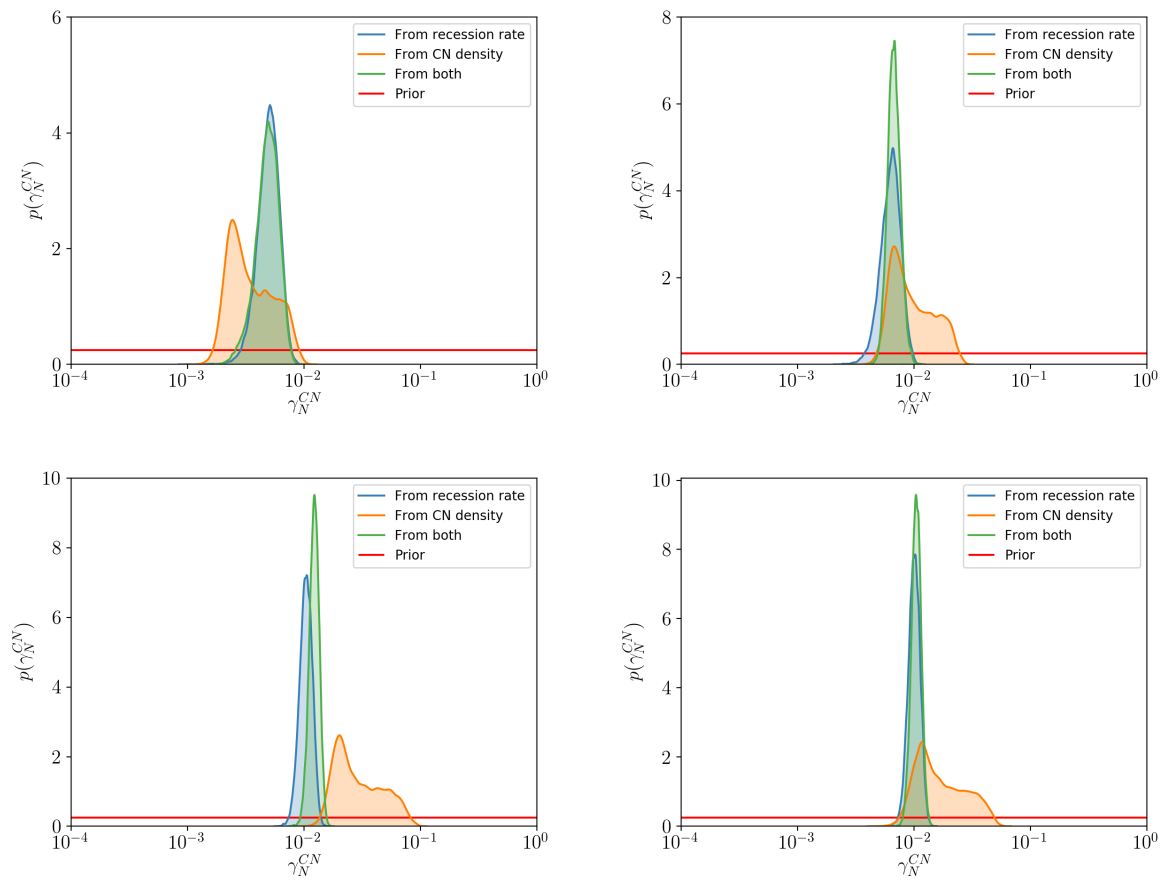

Fig. 3. Marginal posterior distributions of $\gamma_{\mathrm{N}}^{\mathrm{CN}}$ from recession rates, CN densities and both measurements for G4 (upper left), G5 (upper right), G6 (lower left) and G7 (lower right). 


\section{Conclusions}

This contribution reviews some selected experiments and inference methods, centered around the VKI plasma wind tunnel, for catalytic and ablative thermal protection materials. The review showcases the complex, multi-scale phenomena that is atmospheric entry flows. The challenges associated with the understanding of such flows is many fold. In this review, we highlight the intricacies present in selected sets of experimental data and how different models and procedures are used to deterministically retrieve catalytic and ablation coefficients. These methods rely heavily on additional experimental data as well as strong assumptions to fill in the gaps of missing knowledge. We enrich this review by including some of the latest works on Bayesian inference methods for such experimental datasets developed within the UTOPIAE network.

For the case of catalytic materials, the calibration proposed has the impact of improving considerably the inference results by giving consistent and accurate posterior distributions without the need of assuming the value of $\gamma_{\text {ref }}$ for copper. The results show a reduced support and well-defined peaks for both posteriors of $\gamma_{\text {ref }}$ and $\gamma_{\text {TPS }}$. For the ablative material considered, preliminary results show promise in the Bayesian method by taking into account different measurements and combining them together. While the calibrated $\gamma_{\mathrm{N}}^{\mathrm{CN}}$ from recession rates are consistent across the different experimental conditions here explored, the same cannot be said about $\gamma_{\mathrm{N}}^{\mathrm{CN}}$ when calibrated from $\rho_{\mathrm{CN}}$. Further investigations need to be conducted to conclude if it is either an epistemic uncertainty underlying the physical model or biased experimental observations. All in all, it is possible to say that the model parameters can be learned from the experimental conditions considered. Not only that but also these methodologies open the door to future design of experiments to obtain the most accurate coefficients.

\section{Acknowledgments}

This work is fully funded by the European Commission H2020 programme, through the UTOPIAE Marie Curie Innovative Training Network, H2020-MSCAITN-2016, Grant Agreement number 722734.

\section{References}

1. Anderson, J.D.: Hypersonic and high-temperature gas dynamics, second ed. AIAA Education Series (2006)

2. Barbante, P.F.: Accurate and Efficient Modelling of High Temperature Nonequilibrium Air Flows. Ph.D. thesis, ULB/VKI (2001)

3. Barker, M.: On the use of very small pitot-tubes for measuring wind velocity. Proceedings of the Royal Society A: Mathematical, Physical and Engineering Sciences 101 (1922)

4. Bianchi, D., Nasuti, F., Martelli, E.: Navier-stokes simulations of hypersonic flows with coupled graphite ablation. Journal of Spacecraft and Rockets 47, 554-562 (2010) 
5. Bottin, B., Chazot, O., Carbonaro, M., der Haegen, V.V., Paris, S.: The vki plasmatron characteristics and performance. In: Measurement Techniques for High Temperature and Plasma Flows, RTO-EN-8. pp. 6-1, 6-24 (1999)

6. Boubert, P., Vervisch, P.: Cn spectroscopy and physicochemistry in the boundary layer of a c/sic tile in a low pressure nitrogen/carbon dioxide plasma flow. Journal of Chemical Physics 112, 10482-10490 (2000)

7. Chapman, S., Cowling, T.G.: The Mathematical theory of Non-Uniform Gases. Cambridge University Press (1970)

8. Chen, Y.K., Milos, F.: Navier-stokes solutions with finite rate ablation for planetary mission earth reentries. Journal of Spacecraft and Rockets 42 (2005)

9. Chorkendorff, I., Niemantsverdriet, J.W.: Concepts of Modern Catalysis and Kinetics, 3rd Edition. Wiley (2017)

10. Degrez, G., Barbante, P., de la Llave, M., Magin, T.E., Chazot, O.: Determination of the catalytic properties of TPS materials in the VKI ICP facilities. In: European Congress on Computational Methods in Applied Sciences and Engineering ECCOMAS Computational Fluid Dynamics Conference 2001 Swansea, Wales, UK, 4-7 September 2001

11. Duffa, G.: Ablative Thermal Protection Systems Modeling. AIAA Education Series (2013)

12. Ferziger, J.H., Kaper, H.G.: Mathematical Theory of Transport Processes in Gases. North-Holland Publishing Company (1972)

13. Giovangigli, V.: Multicomponent Flow Modeling. Birkhauser (1999)

14. Goulard, R.: On catalytic recombination rates in hypersonic stagnation heat transfer. In: Jet Propulsion. vol. 28 (11), pp. 737-745 (1958)

15. Haario, H., Saksman, E., Tamminen, J.: An adaptive metropolis algorithm. Bernoulli 7, 223-242 (2001)

16. Helber, B.: Material Response Characterization of Low-density Ablators in Atmospheric Entry Plasmas. Ph.D. thesis, Vrije Universiteit Brussel,von Karman Institute for Fluid Dynamics (2016)

17. Helber, B., Turchi, A., Magin, T.E.: Determination of active nitridation reaction efficiency of graphite in inductively coupled plasma flows. Carbon 125, 582-594 (2017)

18. Keenan, J.: Thermo-chemical Ablation of Heat Shields under Earth Re-entry Conditions. Ph.D. thesis, North Carolina State University, Raleigh, North Carolina (1994)

19. Kennedy, M., O'Hagan, A.: Bayesian calibration of computer models. Journal of the Royal Statistical Society: Series B 63, 425-464 (2001)

20. Klomfass, A., Muller, S.: A quasi-onedimensional approach for hypersonic stagnation-point flows. Tech. rep., RWTH Aachen (1996)

21. Kolesnikov, A.F., Tirsky, G.A.: Hydrodynamics equations for partially ionised multicomponent gas mixtures with higher order approximations for transport coefficients. In: Fluid Mechanics-Soviet Research. vol. 13 (4), pp. 70-97 (1984)

22. Kuo, K.K.: Principles of Combustion. John Wiley and Sons, Inc. (2005)

23. Lani, A., Villedieu, N., Bensassi, K., Koloszar, L., Vymazal, M., Yalim, S.M., Panesi, M.: Coolfluid: an open computational platform for multi-physics simulation and research. In: 21st AIAA Computational Fluid Dynamics Conference (20132589)

24. Laub, B., Venkatapathy, E.: Thermal protection system technology and facility needs for demanding future planetary missions. In: Proceedings of international workshop on planetary probe atmospheric entry and descent trajectory analysis and science. pp. $239-247$ (2004) 
25. Madras, N.: Lectures on monte carlo methods. American Mathematical Society (Providence, 2001)

26. Mitchner, M., Kruger, C.H.: Partially Ionized Gases. John Wiley and Sons, Inc (1973)

27. Nelder, J.A., Mead, R.: A simplex method for function minimization. In: Comput. J. vol. 7 (4), p. 308-313 (1965)

28. Panerai, F.: Aerothermochemistry Characterization of Thermal Protection Systems. Ph.D. thesis, Università degli Studi di Perugia,von Karman Institute for Fluid Dynamics (2012)

29. Panerai, F.: Characterization of gas/surface interactions for ceramic matrix composites in high enthalpy, low pressure air flow. Materials Chemistry and Physics 134 (2-3), 597-607 (2012)

30. Panerai, F., Ferguson, J., Lachaud, J., Martin, A., Gasch, M., Mansour, N.: Microtomography based analysis of thermal conductivity, diffusivity and oxidation behavior of rigid and flexible fibrous insulators. International Journal of Heat and Mass Transfer 108, 801-811 (2017)

31. Park, C., Bogdanoff, D.: Shock-tube measurements of nitridation coefficients of solid carbon. Journal of Thermophysics and Heat Transfer 20 (2006)

32. Rasmussen, C.E., Williams, C.K.I.: Gaussian processes for machine learning. the MIT Press (2006)

33. Turchi, A.: A Gas-Surface Interaction Model for the Numerical Study of Rocket Nozzle Flows over Pyrolyzing Ablative Materials. Ph.D. thesis, University of Rome "La Sapienza" (2013)

34. Turchi, A.: Multi-scale Models and Computational Methods for Aerothermodynamics Applications. Ph.D. thesis, École Central Paris, von Karman Institute for Fluid Dynamics (2014)

35. Turchi, A., Bianchi, D., Nasuti, F., Onofri, M.: A numerical approach for the study of the gas-surface interaction in carbon-phenolic solid rocket nozzles. Aerospace Science and Technology 27, 25-31 (2013)

36. Turchi, A., Congedo, P.M., Magin, T.E.: Thermochemical ablation modeling forward uncertainty analysis part i: Numerical methods and effect of model parameters. International Journal of Thermal Sciences 118, 497-509 (2017)

37. Vicenti, W.G., Kruger, C.H.: Introduction to Physical Gas Dynamics. Krieger Pub Co. (1975)

38. Wirgin, A.: Influence of nuisance parameter uncertainty on the retrieval of the thermal conductivity of the macroscopically-homogeneous material within a cylinder from exterior temperature measurements. Applied Mathematical Modelling 39, $5278-5298(2015)$ 